\title{
Randomised vitamin E supplementation and risk of chronic lung disease in the Women's Health Study
}

\author{
Anne H Agler, ${ }^{1}$ Tobias Kurth, ${ }^{2,3,4}$ J Michael Gaziano, ${ }^{2,5,6}$ Julie E Buring, ${ }^{2}$ \\ Patricia A Cassano ${ }^{1}$
}

- Additional data are published online only. To view these files please visit the journal online (http://thorax.bmj.com).

${ }^{1}$ Division of Nutritional Sciences, Cornell University, Ithaca, New York, USA

2Division of Preventive Medicine, Brigham and Women's Hospital, Boston, Massachusetts, USA ${ }^{3}$ INSERM Unit

708-Neuroepidemiology, Paris, France

${ }^{4}$ University Pierre et Marie Curie, Paris, France ${ }^{5}$ Division of Aging, Brigham and Women's Hospital, Boston, Massachusetts, USA

${ }^{6}$ Massachusetts Veteran's Epidemiology, Research and Information Center (MAVERIC) Geriatric Research Education and Clinical Center (GRECC), VA Boston Healthcare System, Boston, Massachusetts, USA

\section{Correspondence to}

Dr P A Cassano, 209 Savage Hall, Cornell University, Ithaca,

NY 14853, USA:

pac6@cornell.edu

Received 6 November 2010 Accepted 16 December 2010 Published Online First 21 January 2011

\section{ABSTRACT}

Background The oxidant/antioxidant balance in lung tissue is hypothesised to contribute to the risk of chronic obstructive pulmonary disease (COPD). Observational studies consistently report higher antioxidant status associated with lower COPD risk, but few randomised studies have been reported.

Methods A post hoc analysis of 38597 women without chronic lung disease at baseline was conducted in the Women's Health Study (WHS) to test the effect of vitamin $\mathrm{E}$ on the risk of incident chronic lung disease. The WHS is a randomised double-blind placebo-controlled factorial trial of vitamin E (600 IU every other day) and aspirin (100 mg every other day) in female health professionals aged $\geq 45$ years. Using Cox proportional hazards models, the effect of randomised vitamin $E$ assignment on self-reported physician-diagnosed chronic lung disease was evaluated.

Results During 10 years of follow-up (376 710 personyears), 760 first occurrences of chronic lung disease were reported in the vitamin $E$ arm compared with 846 in the placebo arm (HR 0.90; $95 \% \mathrm{Cl} 0.81$ to 0.99 ; $p=0.029$ ). This $10 \%$ reduction in the risk of incident chronic lung disease was not modified by cigarette smoking, age, randomised aspirin assignment, multivitamin use or dietary vitamin $\mathrm{E}$ intake (minimum $\mathrm{p}$ for interaction $=0.19$ ). Current cigarette smoking was a strong predictor of chronic lung disease risk (HR 4.17; 95\% Cl 3.70 to 4.70; vs. never smokers).

Conclusions In this large randomised trial, assignment to $600 \mathrm{IU}$ vitamin $\mathrm{E}$ led to a $10 \%$ reduction in the risk of chronic lung disease in women.

\section{INTRODUCTION}

Chronic obstructive pulmonary disease (COPD) is characterised by progressive irreversible airflow limitation and comprises a significant public health burden, with increasing trends in incidence and prevalence. ${ }^{1}$ The prevalence of COPD in the US adult population is $3-4 \%$ and, worldwide, the prevalence of COPD is about $10 \%{ }^{1}{ }^{2}$ COPD was the fifth leading cause of death in the USA in 2001 and is expected to become the third leading cause of death by 2020, largely due to population ageing and increasing cumulative exposure to cigarette smoke, the primary risk factor for COPD. ${ }^{13}$

Factors that may contribute to rising COPD incidence include obesity, dietary patterns, environmental and occupational exposures, and improved diagnostic and screening programmes. ${ }^{1}{ }^{4-6}$ Several lines of evidence support the hypothesis that diet plays a role in the aetiology of COPD. ${ }^{4} 67$ Observational studies of diet or nutritional status biomarkers and randomised trials of diet or nutritional supplements have investigated the relation between antioxidants (notably vitamin $\mathrm{E}$ ) and lung outcomes. Observational studies investigating the association of dietary intake and pulmonary function consistently report that higher intake of nutrients with antioxidant properties is associated with better pulmonary outcomes, but causal inferences are limited by concerns about confounding and other biases. ${ }^{89}$ Studies comparing patients with COPD with healthy individuals report lower plasma and peripheral skeletal muscle vitamin $\mathrm{E}$ ( $\alpha$-tocopherol) concentrations in patients and a lower risk of death from respiratory disease with higher serum $\boldsymbol{\alpha}$-tocopherol concentration, but whether nutrition contributed to the onset of COPD is less clear. ${ }^{10-12}$

Randomised trials of diet change or vitamin $\mathrm{E}$ supplements in clinical populations have reported mixed results. Patients with COPD who increased the intake of antioxidant-rich foods had improved pulmonary function over 3 years while those on usual diets experienced a continuous decline in lung function. ${ }^{13}$ Studies of $\boldsymbol{\alpha}$-tocopherol treatment in patients with COPD have reported mainly negative results, although conclusions are limited by an incomplete understanding of potential to benefit, the short duration of studies and case heterogeneity. ${ }^{14-16}$ Very few large randomised studies of non-diseased individuals have been completed. In the Heart Protection Study (HPS), which included participants with coronary disease, other occlusive arterial disease or diabetes, a post hoc analysis found no effect of vitamin $\mathrm{E}$ supplements on the occurrence of respiratory-related death, on COPD/ asthma hospitalisation rates or on pulmonary function measured by spirometry at the end of the study. ${ }^{17}$ In the Alpha-Tocopherol and Beta-Carotene (ATBC) study, a study of male cigarette smokers, there was no effect of $\alpha$-tocopherol on the incidence of chronic bronchitis or COPD symptoms. ${ }^{16}$

Using data from the Women's Health Study (WHS), a large study of apparently healthy women aged $\geq 45$ years, we tested the hypothesis that supplementation with 600 IU $\alpha$-tocopherol every other day decreases the rate of occurrence of chronic lung disease (CLD).

\section{METHODS}

Additional details are given in the online supplement.

\section{Study design}

The WHS, a randomised double-blind placebocontrolled two-by-two factorial trial, assessed the 
risks and benefits of vitamin E supplements (600 IU every other day; Natural Source Vitamin E Association, Washington, DC, USA) and/or aspirin (100 mg every other day; Bayer AG, Leverkusen, Germany) in the primary prevention of cardiovascular disease and cancer. Full details of the study design are published elsewhere. ${ }^{18}$ The study was registered with http://clinicaltrials. gov/ (NCT00000479).

Eligibility criteria included age $\geq 45$ years; healthcare professional; resident in the USA; no previous history of coronary heart disease, cerebrovascular disease, cancer (except non-melanoma skin cancer) or other major chronic illness; no more than weekly use of vitamins $\mathrm{E}, \mathrm{A}$ or $\beta$-carotene supplements; no history of adverse aspirin effects; less than weekly use of aspirin or non-steroidal anti-inflammatory drugs or willingness to forgo; no use of anticoagulants or corticosteroids. A 3-month placeboonly run-in period identified likely long-term compliers. Of these, 39876 women remained willing and eligible and were randomised into the WHS between April 1993 and January $1996^{18}$

\section{Questionnaire data}

Mailed questionnaires collected baseline data on anthropometric, demographic, lifestyle and clinical characteristics. Follow-up questionnaires, completed twice during the first year and annually thereafter, assessed study supplement compliance, new disease occurrence and diagnosis date, personal characteristics and habits, non-study aspirin, vitamin and non-steroidal anti-inflammatory drug use and side effects. Compliance, defined as taking two-thirds of study supplements, was similar between the active and placebo groups $(78.9 \%$ and $71.6 \%$ at 5 and 10 years, respectively). ${ }^{19}$ Non-trial vitamin E supplement use $\geq 4$ days/month was $10.0 \%$ and $10.9 \%$ at 5 and 10 years, respectively. ${ }^{19}$

\section{CLD ascertainment}

CLD was not a prespecified trial end point. Occurrence of selfreported doctor-diagnosed CLD was ascertained on questionnaires beginning 12 months after study enrolment. A multipart question asked participants "Have you ever been diagnosed by a physician as having any of the following?". Choices included 'other chronic lung disease (eg, emphysema, chronic bronchitis, bronchiectasis)' as well as 'asthma'. For each diagnosis the date of the diagnosis was reported. Thereafter, annual questionnaires asked about diagnoses occurring since the previous questionnaire, including diagnosis date. Incident cases were ascertained to 31 March 2004 (scheduled trial end). Prevalent CLD was defined as CLD diagnosis prior to trial enrolment. Women with prevalent CLD were excluded from the analysis (figure 1).

\section{Statistical analysis}

One thousand two hundred and seventy-nine women reported prevalent CLD (638 in vitamin E group; 641 in placebo group), leaving 38597 participants available for analyses. All analyses followed the intention-to-treat principle. The cumulative incidence of CLD by study arm was assessed using Kaplan-Meier methods and log-rank tests to compare survival curves. Cox proportional hazards models estimated HRs. Further models considered whether the effect of vitamin $\mathrm{E}$ on incident CLD was modified by smoking status, age, body mass index, multivitamin use, alcohol intake, baseline asthma, history of cholesterol levels $\geq 240 \mathrm{mg} / \mathrm{dl}$ or randomised aspirin assignment. Effect modification was tested for statistical significance using likelihood ratio tests comparing models with and without interaction terms.

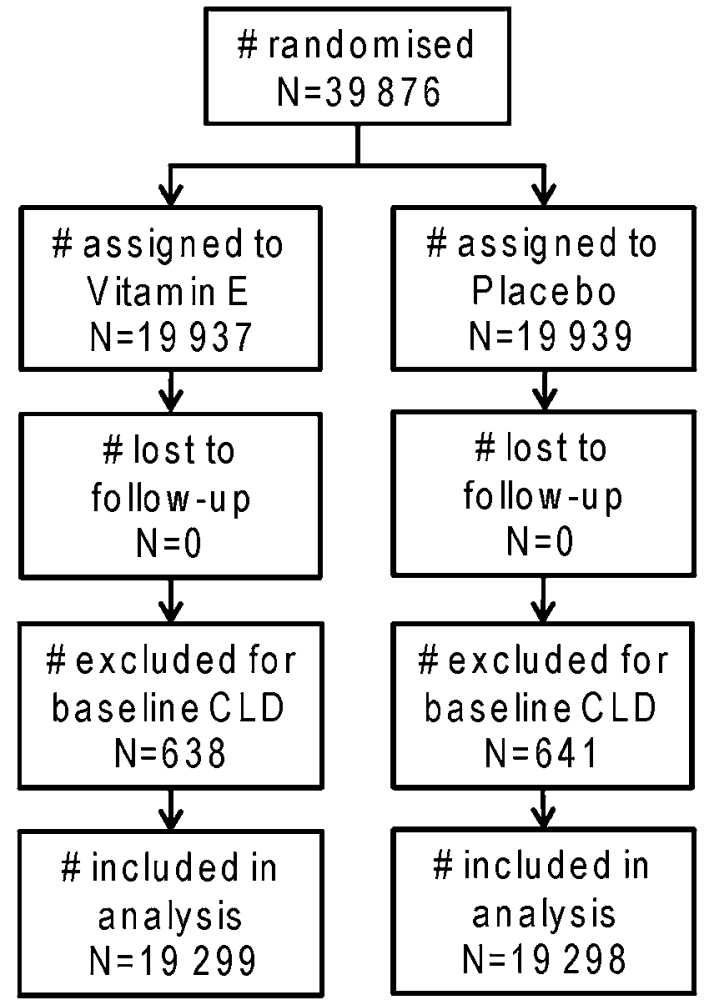

Figure 1 Flow diagram of the vitamin E component of the Women's Health Study chronic lung disease (CLD) analysis.

Data management and analyses were completed using SAS (SAS Institute Inc).

\section{RESULTS}

The baseline characteristics of the 38597 participants, summarised in table 1, were balanced between the vitamin $\mathrm{E}$ and placebo arms. Thus, participants in both arms were similar in age, smoking, body mass index, multivitamin use, dietary intake of vitamin E, alcohol intake, history of asthma diagnosis and percentage with cholesterol $\geq 240 \mathrm{mg} / \mathrm{dl}$ (table 1 ). The mean age of study participants was 54.5 years, and women were followed on average for 9.8 years (376710 person-years; 188578 person-years in the vitamin E arm, 188132 person-years in the placebo arm).

Participants reported 1606 new diagnoses of CLD, corresponding to a cumulative incidence of $4.2 \%$. Participants in the vitamin $\mathrm{E}$ arm reported 760 incident CLD diagnoses (cumulative incidence $3.9 \%$ ) compared with 846 diagnoses in the placebo arm (cumulative incidence 4.4\%; figure 2), corresponding to a statistically significant $10 \%$ reduction in risk in participants randomised to receive vitamin $\mathrm{E}$ supplements (HR 0.90; $95 \% \mathrm{CI}$ 0.81 to $0.99 ; \mathrm{p}=0.029)$. Comparing the cumulative CLD incidence by year of follow-up in the vitamin $\mathrm{E}$ and placebo groups (figure 3), the curves separate at about 1.5 years of study and continue to diverge until about 5 years of supplementation, maintaining a consistent separation thereafter. In contrast, the aspirin intervention had little or no association with risk of CLD (HR 0.98 ; $95 \%$ CI 0.89 to 1.08 ).

Cigarette smoking had a strong association with CLD incidence (current smoker vs never smoker: HR 4.17; 95\% CI 3.70 to 4.70; $\mathrm{p}<0.0001$ ). In addition, other known COPD risk factors were positively associated with the CLD outcome: older age at randomisation (age $\geq 65$ years vs $<55$ years; HR $2.38 ; 95 \% \mathrm{CI}$ 2.07 to 2.73 ; $\mathrm{p}<0.0001$ ), obesity (BMI $\geq 30.0$ vs $<25.0$; HR 1.60 ; $95 \%$ CI 1.41 to $1.81 ; \mathrm{p}<0.0001)$, asthma diagnosis prior to 
Table 1 Baseline characteristics of participants in the Women's Health Study by vitamin E randomisation

\begin{tabular}{|c|c|c|}
\hline Characteristic & $\begin{array}{l}\text { Vitamin } E(N=19299) * \\
N(\%) \neq\end{array}$ & $\begin{array}{l}\text { Placebo }(N=19298) \dagger \\
N(\%) \neq\end{array}$ \\
\hline \multicolumn{3}{|l|}{ Demographic/lifestyle } \\
\hline Age, years $\ddagger$ & $54.5(7.0)$ & $54.6(7.0)$ \\
\hline$<55$ & $11714(60.7)$ & $11679(60.5)$ \\
\hline $55-64$ & $5654(29.3)$ & $5677(29.4)$ \\
\hline$\geq 65$ & $1931(10.0)$ & $1942(10.1)$ \\
\hline \multicolumn{3}{|l|}{ Cigarette smoking } \\
\hline Current & $2434(12.6)$ & $2491(12.9)$ \\
\hline Past & $6937(36.0)$ & $6823(35.4)$ \\
\hline Never & 9909 (51.4) & $9968(51.7)$ \\
\hline Average duration, years $\ddagger \S$ & $18.8(12.5)$ & $18.9(12.6)$ \\
\hline Body mass index, $\mathrm{kg} / \mathrm{m}^{2} \ddagger$ & $26.0(5.0)$ & $26.0(5.0)$ \\
\hline$<25.0$ & $9598(50.8)$ & $9670(51.1)$ \\
\hline $25.0-<30.0$ & $5880(31.1)$ & $5837(30.8)$ \\
\hline$\geq 30.0$ & 3404 (18.0) & $3411(18.0)$ \\
\hline \multicolumn{3}{|l|}{ Nutrition } \\
\hline \multicolumn{3}{|l|}{ Multivitamin use } \\
\hline Never & $2521(13.2)$ & $2553(13.4)$ \\
\hline Past only & $10927(57.4)$ & $10982(57.7)$ \\
\hline Current & 5574 (29.3) & $5499(28.9)$ \\
\hline \multicolumn{3}{|l|}{ Vitamin E intake, mg/day } \\
\hline Diet onlył & $6.6(5.0)$ & $6.6(5.3)$ \\
\hline Diet+supplements $\ddagger$ & $63.2(143.0)$ & $62.6(140.9)$ \\
\hline \multicolumn{3}{|l|}{ Alcohol intake } \\
\hline Rare/never & $8743(45.3)$ & $8590(44.5)$ \\
\hline $1-3 /$ month & $2531(13.1)$ & $2553(13.2)$ \\
\hline $1-6 /$ week & $6048(31.4)$ & $6194(32.1)$ \\
\hline $1+/$ day & $1970(10.2)$ & $1959(10.2)$ \\
\hline \multicolumn{3}{|l|}{ Medical conditions } \\
\hline Asthma diagnosis & $1104(5.7)$ & $1105(5.7)$ \\
\hline Cholesterol $\geq 240 \mathrm{mg} / \mathrm{dl}$ & $5615(29.1)$ & $5688(29.5)$ \\
\hline Study aspirin assignment & $9638(49.9)$ & $9654(50.0)$ \\
\hline \multicolumn{3}{|c|}{$\begin{array}{l}{ }^{*} \mathrm{~N} \text { total for each characteristic ranges from } 18882 \text { to } 19299 \text {, given missing data in some } \\
\text { variables. } \\
\dagger \mathrm{N} \text { total for each characteristic ranges from } 18918 \text { to } 19298 \text {, given missing data in some } \\
\text { variables. } \\
\text { †Continuous variables are presented as mean (SD). } \\
\S \text { Average smoking duration for current and past smokers only. }\end{array}$} \\
\hline
\end{tabular}

randomisation (HR 1.94; 95\% CI 1.65 to 2.28; $\mathrm{p}<0.0001$ ) and hypercholesterolaemia (HR 1.42; 95\% CI 1.28 to 1.57 ; $\mathrm{p}<0.0001$ ).

There was no statistical evidence that the effect of randomised vitamin $\mathrm{E}$ assignment on CLD risk was modified by age $(p=0.86)$, smoking status $(p=0.96)$, body mass index $(p=0.25)$, multivitamin use $(p=0.67)$, baseline asthma history $(p=0.89)$, cholesterol $\geq 240 \mathrm{mg} / \mathrm{dl}(\mathrm{p}=0.84)$ or by study aspirin assignment $(p=0.19)$ (figure 3). Alcohol intake was borderline statistically significant $(p=0.054)$ as a modifier of the effect of vitamin $E$ on CLD, and women consuming one or more alcoholic drinks per day had the strongest vitamin $\mathrm{E}$ protective effect. In additional analyses there was no evidence of effect modification by race, exercise frequency, hypertension and baseline dietary intake of either vitamin $\mathrm{E}$ or $\mathrm{C}$. For all models, controlling for randomised aspirin assignment did not alter the effect of vitamin $\mathrm{E}$ supplement assignment on CLD risk. An additional sensitivity analysis was conducted, censoring women who reported incident asthma from the CLD analysis; the association of vitamin E was similar with a $9 \%$ reduction in risk of CLD (HR 0.91; 95\% CI 0.81 to 1.03).

\section{DISCUSSION}

In this large randomised double-blind placebo-controlled trial in apparently healthy women, $600 \mathrm{IU}$ vitamin $\mathrm{E}$ on alternate days reduced the risk of self-reported newly diagnosed CLD by approximately $10 \%$. There was no statistically significant difference in the magnitude of the effect of vitamin $\mathrm{E}$ by age, smoking status, randomised aspirin assignment, multivitamin use or asthma history, and the protective effect was slightly stronger in women consuming $>1$ alcoholic drink/day. Randomised assignment to aspirin had no association with the risk of CLD.

Two previous randomised trials investigated vitamin $\mathrm{E}$ supplementation in relation to lung outcomes and both reported no effect of intervention. ${ }^{16} 17$ In the HPS, 20536 adults aged 40-80 years with prevalent coronary artery disease were randomised to a combined intervention of $600 \mathrm{IU}$ vitamin E, $250 \mathrm{mg}$ vitamin $\mathrm{C}$ and $20 \mathrm{mg} \beta$-carotene or placebo daily for 5 years. ${ }^{17}$ The HPS differed from the WHS in important ways. The HPS enrolled participants with clinically diagnosed coronary artery disease, occlusive arterial disease or diabetes and $75 \%$ of the participants were men ${ }^{17}$ while the WHS comprised women without a history of cardiovascular disease. Study duration (5 years in the HPS vs 10 years in the WHS) and supplement formulation (combined supplement in the HPS vs vitamin E alone in the WHS) also differed. Finally, the outcomes in the HPS (pulmonary function measured by spirometry, death due to respiratory illness and COPD or asthma-related hospitalisations) differed from the WHS which investigated the incidence of a CLD diagnosis. ${ }^{17}$

In the ATBC study, 29133 male cigarette smokers aged 50-69 years were randomised to receive $50 \mathrm{mg}$ vitamin $\mathrm{E}$ and/or $20 \mathrm{mg} \beta$-carotene or placebo daily for 4 years. ${ }^{16}$ The ATBC differed substantially from the WHS in the supplement studied, trial duration and the population studied. ${ }^{16}$ The ATBC reported no effect of vitamin $\mathrm{E}$ on COPD-related symptoms, a substantially different end point from the incidence of CLD diagnosis.

The strengths of the WHS include the large number of participants, the large number of self-reported doctor-diagnosed CLD outcomes, high adherence rate and high follow-up rate. CLD is associated with ageing, so the minimum age required for study enrolment ( 45 years) yielded a population at risk for incident CLD.

Several limitations deserve mention. While the size of this trial was adequate to detect a statistically significant small to moderate effect of vitamin $\mathrm{E}$ supplementation on incident CLD, the trial was not specifically designed to test the studied hypothesis. Thus, outcome ascertainment was based solely on self-reported doctor diagnosis, a concern that is partly mitigated by the fact that participants were female health professionals. A validation study of self-reported COPD outcomes in female nurses found that $78 \%$ of self-reported cases of COPD were confirmed by medical record review, which suggests that selfreport of lung disease by the female health professionals comprising the WHS is likely to have excellent validity. ${ }^{20}$ While the question about outcome occurrence included bronchiectasis, the low incidence of bronchiectasis in this age range leads to the reasonable assumption that most occurrences reported refer to COPD. Current cigarette smoking was a strong predictor of CLD in these data, providing evidence of face validity for the outcome ascertainment. Finally, if the outcome is misclassified (either by undercounting cases or by including false positives), the misclassification is likely to affect both arms of the trial equally and the HR may therefore be an underestimate of the true effect size.

\section{Outcome definition}

The complexity of airway disease phenotypes raises substantial concern about misdiagnosis of COPD and asthma, particularly 


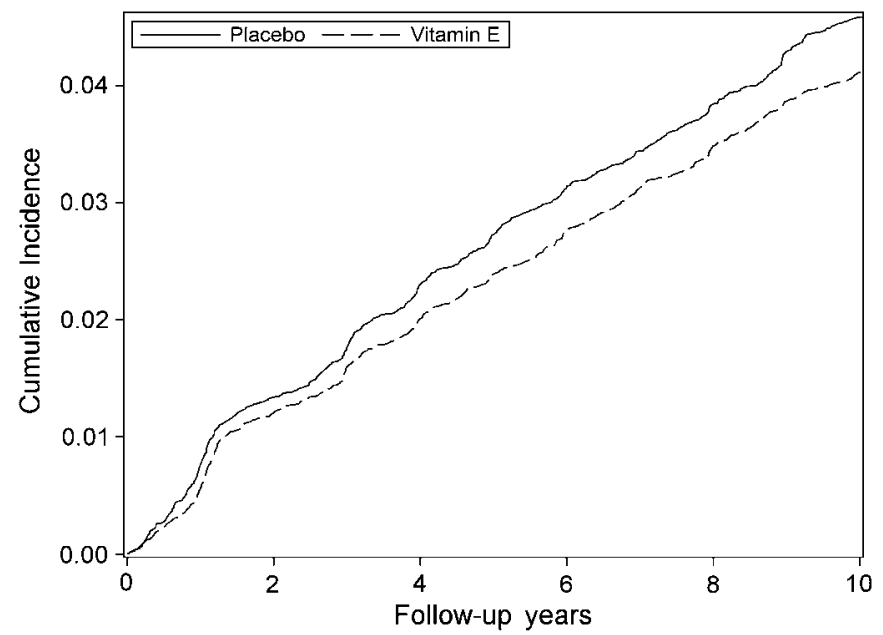

Figure 2 Cumulative incidence of chronic lung disease during the randomised component of the Women's Health Study.

in women. ${ }^{21}$ To address the possibility that women reporting a new asthma diagnosis actually had COPD, a sensitivity analysis was conducted. When women reporting incident asthma were excluded from the analysis, vitamin $\mathrm{E}$ was associated with a $9 \%$ reduction in the risk of CLD (HR 0.91; 95\% CI 0.81 to 1.03), similar to the findings in the full study group. There was little or no effect of vitamin E supplementation on incident selfreported doctor-diagnosed asthma (HR 0.99; 95\% CI 0.90 to 1.08; $\mathrm{p}=0.83$ ). Kurth et al ${ }^{22}$ investigated the effect of randomised aspirin assignment on the risk of incident adult-onset asthma in the WHS; women in the aspirin arm had a $10 \%$ lower risk of incident asthma than those in the placebo arm. When women developing COPD over follow-up were censored from the analysis, the findings were similar. The effect of aspirin on incident asthma and the lack of effect of aspirin on incident CLD supports the notion of differentiation in the self-reported diagnoses.

\section{Proposed mechanisms}

Previous studies have documented the presence of vitamin $\mathrm{E}$ in the lung compartment and the mechanisms of delivery of vitamin $\mathrm{E}$ to alveolar type II cells in the lung. ${ }^{23}$ Vitamin $\mathrm{E}$ transport to the type II cells is hypothesised to occur via highdensity lipoproteins because type II cells have no physical contact with plasma and interact only with interstitial fluid lipoproteins, which are predominantly HDL lipoproteins. ${ }^{23}$ Thus, the concentration of HDL cholesterol in the plasma and in the interstitial fluid predicts the amount of vitamin E available to the lung compartment to combat oxidative stress. Previous studies have reported that HDL cholesterol and apolipoprotein A-I levels are positively associated with forced expiratory volume in $1 \mathrm{~s}$, even after adjusting for serum antioxidant concentrations, a finding that may reflect the delivery of vitamin E to lung tissues. ${ }^{24}$ Thus, a higher HDL cholesterol level is hypothesised to deliver a greater effective dose of vitamin $\mathrm{E}$ to the lung compartment.

Given that HDL cholesterol levels are $20-25 \%$ higher in women than in men of all age groups, differences in the biologically effective dose of vitamin $\mathrm{E}$ may contribute to the difference in findings between women in the WHS and the
Figure 3 Effect modification of the vitamin $\mathrm{E} /$ chronic lung disease effect in the Women's Health Study. BMI, body mass index.

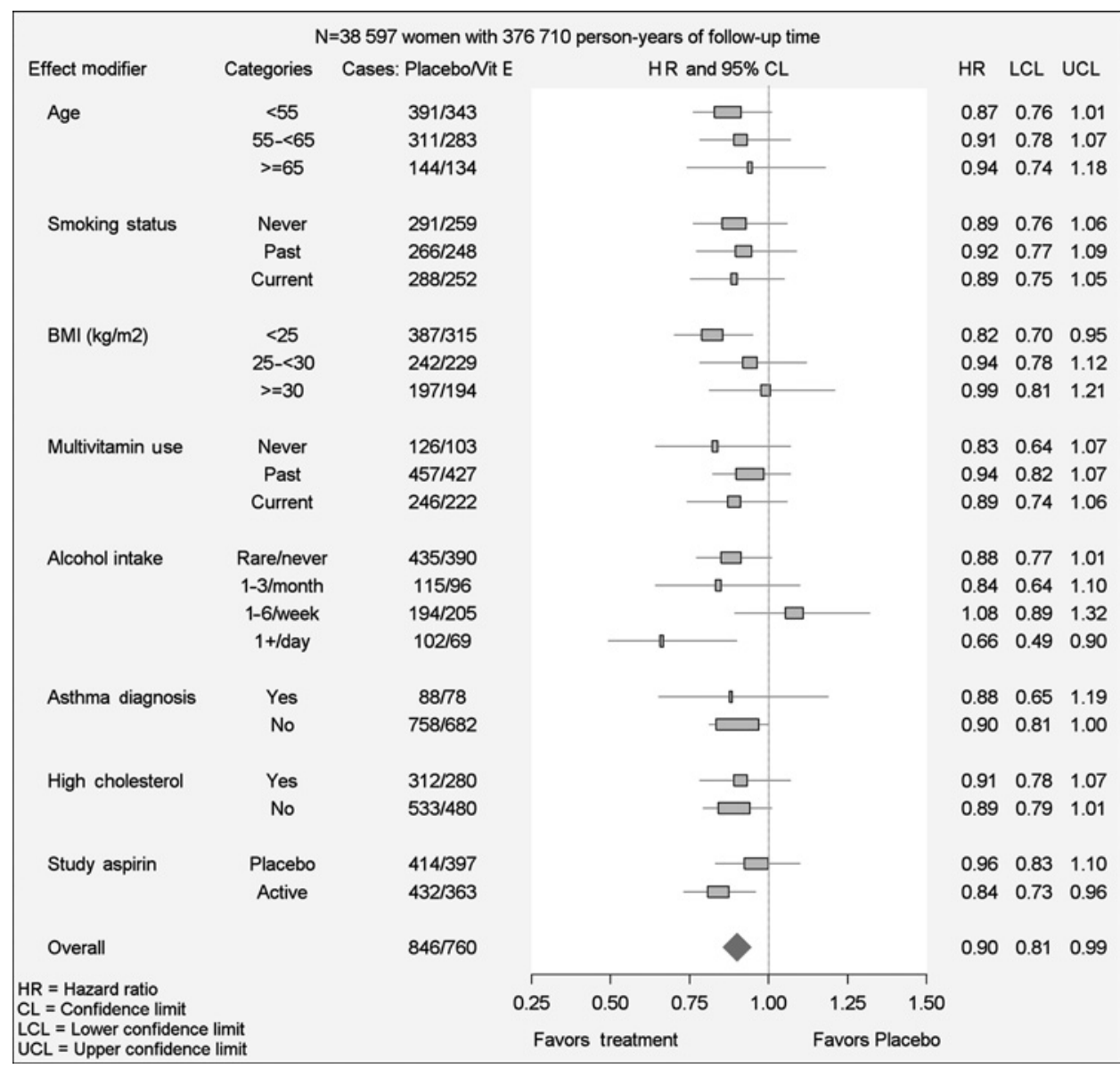


predominantly male participants in the HPS and ATBC. ${ }^{25}$ Sexrelated differences in the effect of vitamin E supplementation on all-cause mortality have been reported, with stronger protective effects of low-dose vitamin E supplementation evident in study populations comprised of $\geq 75 \%$ women. $^{26}$

Alcohol intake is proposed to reduce the risk of cardiovascular disease by raising HDL cholesterol levels, and a threshold of $\geq 1$ alcoholic drink per day is associated with both higher HDL cholesterol levels and attenuation of the risk of cardiovascular disease. $^{27} 28$ Given the important role of HDL cholesterol in transporting vitamin $\mathrm{E}$ to the lung, exploratory analyses considered alcohol intake as a modifier of the effect of vitamin $\mathrm{E}$ on CLD. Among variables considered as possible effect modifiers, alcohol intake was marginally statistically significant $(\mathrm{p}=0.054)$, and the preventive effect of vitamin $\mathrm{E}$ on CLD was greatest in women consuming the highest level of alcohol ( $\geq 1 \mathrm{drink} /$ day). These preliminary findings are consistent with the hypothesis that delivery of vitamin E to lung tissue may vary by plasma levels of HDL cholesterol.

Under our hypothesis, the effect of antioxidant supplementation was expected to be stronger in participants with a higher oxidative burden-for example, current cigarette smokers. Contrary to expectation, there was no evidence of effect modification by smoking. If supplementation with vitamin $\mathrm{E}$ acts through other systemic mechanisms-for example by improving immune system function-then a general effect of vitamin $\mathrm{E}$ would be supported. ${ }^{29}$

\section{Efficacy and safety considerations}

There has been substantial discussion of the efficacy and safety of vitamin $\mathrm{E}$ supplementation in the scientific literature. ${ }^{30}$ Potential harmful effects include an increased risk of allcause mortality, susceptibility to bleeding and haemorrhagic stroke. $^{31-33}$ The meta-analysis linking high-dose vitamin E supplementation to increased risk of mortality, however, has been criticised for its methodology and a recent paper suggested that vitamin $\mathrm{E}$ has beneficial effects on the risk of ischaemic stroke. $^{33-35}$ Thus, the design of future vitamin E supplementation trials must carefully consider information about risks and benefits, and recommendations may need to be tailored to specific populations.

\section{CONCLUSION}

The WHS comprised female health professionals aged $>45$ years, the majority of whom were of European descent. Healthy women taking 600 IU vitamin E supplements every other day were $10 \%$ less likely to report a new CLD diagnosis during the study period. Any decisions about use of vitamin $\mathrm{E}$ as a preventive must consider information about vitamin E-associated risks and bioavailability. ${ }^{22} 26 \quad 30 \quad 32-34$ Given that there are few prevention strategies for emphysema and chronic bronchitis, further study of vitamin $\mathrm{E}$ in relation to COPD is of public health interest.

Acknowledgements The authors thank the participants in the Women's Health Study for their outstanding commitment and cooperation, the entire staff of the Women's Health Study for their expert assistance and the Division of Preventive Medicine, Brigham and Women's Hospital, Harvard Medical School, Boston, Massachusetts, USA.

Funding This study was funded by grants from the National Institutes for Health, USA, grants NIH HL071022 (PAC); NIH HL043851 and CA47988 (Women's Health Study).

Competing interests None.
Ethics approval This study was conducted with the approval of the Brigham and Women's Hospital and Cornell University (for analyses in this study of previously collected data). The Women's Health Study was approved by the institutional review board of Brigham and Women's Hospital and monitored by an external data and safety monitoring board.

Contributors Conception and design of Women's Health Study: JEB, JMG; conception of research question: PAC, AHA; analysis and interpretation: AHA, PAC, TK; writing and editing: AHA, PAC, TK, JEB, JMG.

Provenance and peer review Not commissioned; externally peer reviewed.

\section{REFERENCES}

1. Lopez AD, Shibuya K, Rao C, et al. Chronic obstructive pulmonary disease: current burden and future projections. Eur Respir J 2006:27:397-412.

2. Buist AS, Vollmer WM, McBurnie MA. Worldwide burden of COPD in high- and lowincome countries. Part I. The burden of obstructive lung disease (BOLD) initiative. Int J Tuberc Lung Dis 2008:12:703-8.

3. Lopez AD, Murray CC. The global burden of disease, 1990-2020. Nat Med 1998:4:1241-3.

4. Varraso R, Fung TT, Barr RG, et al. Prospective study of dietary patterns and chronic obstructive pulmonary disease among US women. Am J Clin Nutr 2007:86:488-95.

5. Sood A, Qualls C, Arynchyn A, et al. Obesity-asthma association: is it explained by systemic oxidant stress? Chest 2009;108:744-53.

6. Smit HA, Grievink L, Tabak C. Dietary influences on chronic obstructive lung disease and asthma: a review of the epidemiological evidence. Proc Nutr Soc 1999:58:309-19.

7. Romieu I, Trenga C. Diet and obstructive lung diseases. Epidemiol Rev 2001;23:268-87.

8. Hu G, Cassano PA. Antioxidant nutrients and pulmonary function: the Third National Health and Nutrition Examination Survey (NHANES III). Am J Epidemiol 2000;151:975-81.

9. Grievink L, Smit HA, Ocke MC, et al. Dietary intake of antioxidant (pro)-vitamins, respiratory symptoms and pulmonary function: the MORGEN study. Thorax 1998;53:166-71.

10. McKeever TM, Lewis SA, Smit HA, et al. A multivariate analysis of serum nutrient levels and lung function. Respir Res 2008;9:67-76.

11. Gosker HR, Bast A, Haenen GR, et al. Altered antioxidant status in peripheral skeletal muscle of patients with COPD. Respir Med 2005:99:118-25.

12. Wright ME, Lawson KA, Weinstein SJ, et al. Higher baseline serum concentrations of vitamin $\mathrm{E}$ are associated with lower total and cause-specific mortality in the AlphaTocopherol, Beta-Carotene Cancer Prevention Study. Am J Clin Nutr 2006;84:1200-7

13. Keranis $\mathbf{E}$, Makris D, Rodopoulou $\mathrm{P}$, et al. Impact of dietary shift to higher antioxidant foods in COPD: a randomized trial. Eur Respir J 2010;36:774-80.

14. Daga MK, Chhabra R, Sharma B, et al. Effects of exogenous vitamin E supplementation on the levels of oxidants and antioxidants in chronic obstructive pulmonary disease. J Biosci 2003;28:7-11.

15. Nadeem A, Raj HG, Chhabra SK. Effect of vitamin E supplementation with standard treatment on oxidant-antioxidant status in chronic obstructive pulmonary disease. Indian J Med Res 2008;128:705-11.

16. Rautalahti M, Virtamo J, Haukka J, et al. The effect of alpha-tocopherol and betacarotene supplementation on COPD symptoms. Am J Respir Crit Care Med 1997; 156:1447-52

17. Heart Protection Study Collaborative Group. MRC/BHF Heart Protection Study of antioxidant vitamin supplementation in 20,536 high-risk individuals: a randomised placebo-controlled trial. Lancet 2002;360:23-33.

18. Rexrode KM, Lee IM, Cook NR, et al. Baseline characteristics of participants in the Women's Health Study. J Womens Health Gend Based Med 2000;9:19-27.

19. Lee IM, Cook NR, Gaziano JM, et al. Vitamin E in the primary prevention of cardiovascular disease and cancer: the Women's Health Study: a randomized controlled trial. JAMA 2005;294:56-65

20. Barr RG, Herbstman J, Speizer FE, et al. Validation of self-reported chronic obstructive pulmonary disease in a cohort study of nurses. Am J Epidemiol 2002;155:965-71.

21. Tinkelman DG, Price DB, Nordyke RJ, et al. Misdiagnosis of COPD and asthma in primary care patients 40 years of age and over. J Asthma 2006;43:75-80.

22. Kurth T, Barr RG, Gaziano JM, et al. Randomised aspirin assignment and risk of adult onset asthma in the Women's Health Study. Thorax 2008;63:514-18.

23. Kolleck I, Sinha P, Rustow B. Vitamin E as an antioxidant of the lung: mechanisms of vitamin E delivery to alveolar type II cells. Am J Respir Crit Care Med 2002;166 S62-6.

24. Cirillo DJ, Agrawal Y, Cassano PA. Lipids and pulmonary function in the Third National Health and Nutrition Examination Survey. Am J Epidemiol 2002:155:842-8.

25. Cheung BM, Li M, Ong KL, et al. High density lipoprotein-cholesterol levels increase with age in American women but not in Hong Kong Chinese women. Clin Endocrinol 2009; 70:561-8.

26. Gerss J. The association of vitamin E supplementation and mortality - finally consistent results of statistical analysis. RE: The questionable association of vitamin $E$ supplementation and mortality - inconsistent results of different meta-analytic approaches. Cell Mol Biol 2010;56:0L1266-7. 
27. De Oliveira E Silva ER, Foster D, McGee HM et al. Alcohol consumption raises $\mathrm{HDL}$ cholesterol levels by increasing the transport rate of apolipoproteins $\mathrm{A}-\mathrm{I}$ and $\mathrm{A}-\mathrm{II}$. Circulation 2000;102:2347-52.

28. Foerster M, Marques-Vidal P, Gmel G, et al. Alcohol drinking and cardiovascular risk in a population with high mean alcohol consumption. Am J Cardiol 2009;103:361-8.

29. Meydani SN, Han SN, Wu D. Vitamin E and immune response in the aged: molecular mechanisms and clinical implications. Immunol Rev 2005:205:269-84.

30. Bell SJ, Grochoski GT. How safe is vitamin E supplementation? Crit Rev Food Sci Nutr 2008;48:760-74.
31. Miller ER III, Pastor-Barriuso R, Dalal D et al. Meta-analysis: high-dosage vitamin E supplementation may increase all-cause mortality. Ann Intern Med 2005;142:37-46.

32. Violi F, Pignatelli P, Basili S. Nutrition, supplements, and vitamins in platelet function and bleeding. Circulation 2010:121:1033-44.

33. Shurks M, Glynn RJ, Rist PM, et al. Effects of vitamin E on stroke subtypes: metaanalysis of randomised controlled trials. BMJ 2010;341:c5702.

34. Gerss J, Kopcke W. The questionable association of vitamin E supplementation and mortality-inconsistent results of different meta-analytic approaches. Cell Mol Biol 2009:55(Suppl):OL1111-20.

35. Berry D, Wathen JK, Newell M. Bayesian model averaging in meta-analysis: vitamin E supplementation and mortality. Clin Trials 2009;6:28-41.

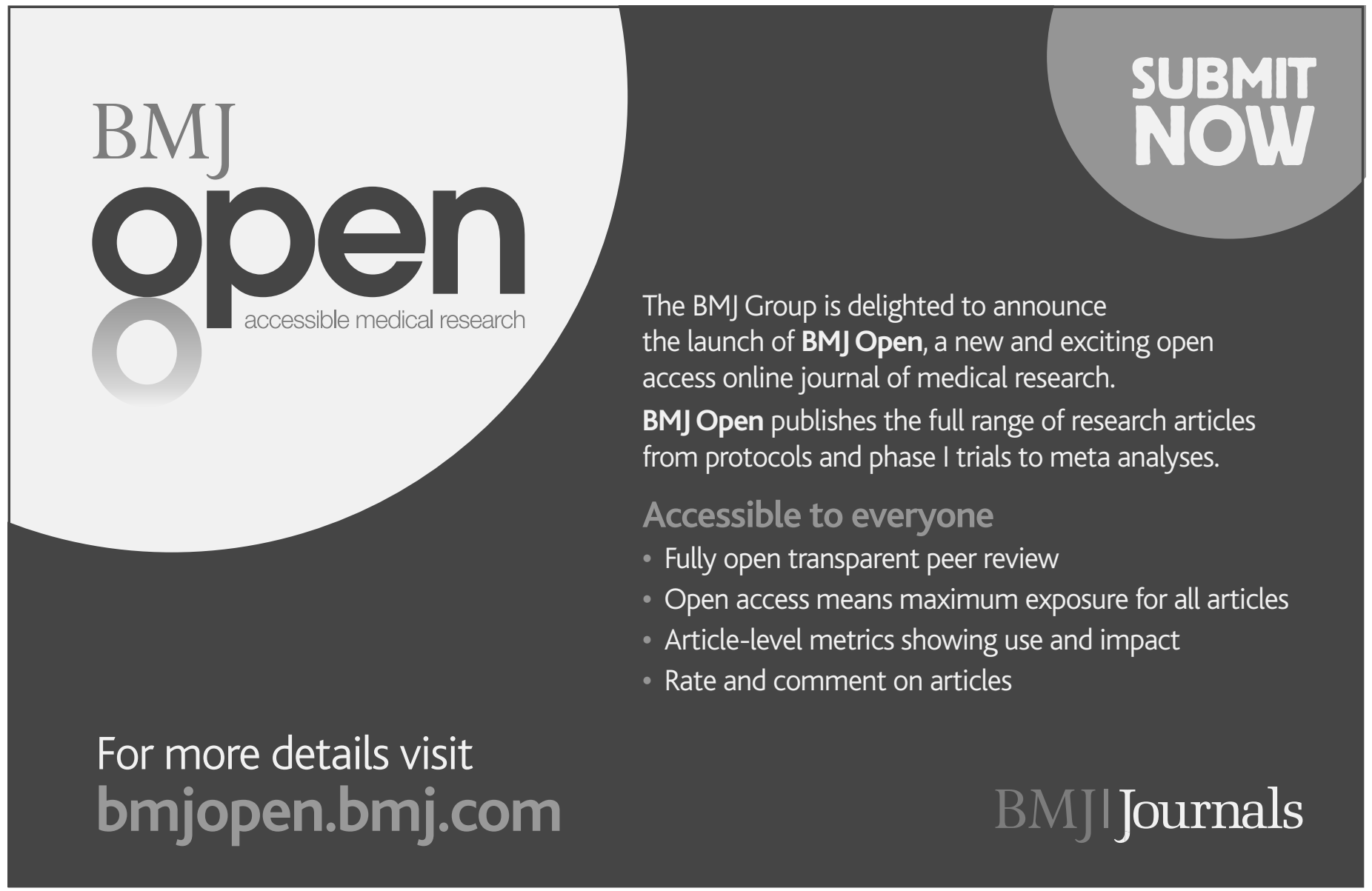

